\title{
Tratamiento del Shock Séptico sin Drogas Vasopresoras
}

\author{
Doctor Jorge E. Zuñiga \\ Profesor Asociado Facultad de Medicina \\ Dpto. de Obstetricia y Ginecología \\ Universidad del Valle \\ Jefe de Servicio Ginecología
}

\section{INTRODUCCION}

Dada la alta mortalidad y morbilidad de esta entidad en nuestro medio, el hecho de ser bastante común, principalmente como consecuencia del aborto provocado, en la última decada nos hemos interesado por su estudio y tratamiento, basados fundamentalmente en los nuevos planteamientos fisiopatológicos que dan énfasis en el mantenimiento de una adecuada Perfusión Tisular, bloqueandó la vaso-constricción y reem plazando la pérdida de líquidos para obtener una Volemia Normal.

En nuestro plan de tratamiento se ha utilizado la Clorpromazina, que se considera como un agente que reduce el tono vascular por mecanismos no bien conocidos (9) (31) (19)

No utilizamos otras drogas de finalidad más lógica, como las del grupo de los Bloqueadores Adrenérgicos, que previenen la acción Vaso-constrictora como la Fenoxybenzamina (36) (Dibenzylina), por la dificultad de conseguirlos en nuestro país; próximamente iniciaremos un estudio con la Orciprenalina, droga que ha demostrado poseer un poder estimulante Beta- Adrenérgico y por lo tanto bloqueador de la vasoconstricción y estimulante de la vasodilatación cuyo uso, desde el punto de vista teórico tiene fundamentos mucho más lógicos que la droga utilizada en el estudio que estamos publicando.

En el presente estudio se hace una reseña histórica del problema clínico, se dá una

definición del mismo; se analizan factores etiológicos haciendo consideraciones fisiopatológicas.

Se expone un plan de tratamien to de acuerdo con consideraciones fisiopatológicas; se investigan los resultados en 15 casos con problemas de infección pélvica de diverso origen, haciendo un análisis cuidadoso de cada uno y mostrando los resultados obtenidos; al final se dán las conclusiones a las que se llegó por el estudio de los casos presentados y la revisión de una buena cantidad de trabajos ya publicados.

\section{TRATAMIENTO DEL SHOCK SEPTICO SIN VASOPRESORES}

Dados los conocimientos modernos sobre la naturaleza y la fisiopatología del Shock, se han hecho notables cambios en su profilaxis y tratamiento, lo que ha llevado a reducir en una forma altamente significativa la mortalidad por este Sindrome. A manera de introducción, hacemos un resumen sobre los siguientes aspectos:

1. Reseña Histórica.

2. Definición

3. Etiología - Factores precipitantes.

4. Mecanismo Fisiopatológico (Teorías microcirculación).

5. Curso Clínico

6. Tratamiento.

7. Análisis de quice casos tratados según programa de manejo. 


\section{RESEÑA HISTORICA}

El Shock es un Sindrome que se conoce desde hace casi 2000 años.

En tiempos del nacimiento de Cristo, Celso había escrito: "Cuando la sangre no llega al corazón en cantidad suficiente, el pulso es débil, la piel está muy pálida, hay por todo el cuerpo un sudor frío y fétido y enfriamiento de las extremidades; la muerte sobreviene rápidamente".

Esto, aún en nuestros días, sigue siendo una admirable descripción clínica.

El Sindrome de Shock Séptico fué reconocido y descrito por Leannec en 1831. El diagnóstico fué hecho cuando una paciente con infección por gérmenes gramnegativos, tuvo colapso-vasomotor sin pérdida significativa de Sangre.

Recientemente se le ha descrito como causado por invasión de gérmenes gram-positivos. (1)

\section{DEFINICION}

\section{EL SHOCK ES UN SINDROME CA-} RACTERIZADO POR:

Rendimiento cardíaco inadecuado.

Baja en volumen circulatorio efectivo.

Falla circulatoria periférica; Hipotensión severa.

Oliguria (menos de $15 \mathrm{cc}$. de orina por hora).

Transtornos mentales (como obnubilación y desorientación).

Si no se corrige el deterioro progresivo de la circulación, se desarrolla inadecuada perfusión de los tejidos y muerte celular. Cualquiera que sea el mecanismo, se dice que el Shock está presente cuando falla la presión sanguínea para retornar a lo normal sin terapia de soporte.

Hace tiempo se clasificaba el tipo de Shock según el probable mecanismo desencadenante: Shock Cardiogénico del infarto del miocardio; Shock Neurogénico por trauma del sistema nervioso; Shock Hemorrágico, por pérdida significativa de sangre. Pero según parece el sindrome se debe a un, mecanismo final común, con el cual el or- ganismo responde a diversas agresiones. $\mathrm{Pa}$ rece ser que en todos los tipos de Shock el mecanismo común resultante es una inadecuada perfusión tisular.

El Shock Hemorrágico es el tipo más común encontrado en pacientes de Obstetricia y Ginecología y puede resultar de hemorragias internas o externas.

La causa es generalmente obvia y con control de la hemorragia y reemplazo de sangre perdida, el resultado es muy satisfactorio.

El Shock no hemorrágico y especialmente el Shock Bacteriano por gérmenes gramnegativos es un problema mucho más complejo. (2)

\section{ETIOLOGIA}

Factores Precipitantes:

Puede ser el resultado de una combinación de factores. Fine y colaboradores demostraron que las Bacterias juegan papel importante en el Shock. (28).

Hay invasión en la circulación de productos Bacterianos como las Endotoxinas que son constituyentes lipopolisacáridos de la pared celular que producen falla vascular profunda.

Recientemente se ha descrito el Shock causado por invasión de gérmenes grampositivos que liberan Exotoxinas y sustancias vasoactivas como:
Histamina
Serotonina
Catecolaminas
Polipéptidos

\section{MECANISMO FISIOPATOLOGICO \\ (23) (49) (45) (48) (25)}

\section{Teorías - Microcirculación}

Perfusión Capilar inadecuada (3)

Las arteriolas rara vez están todas abiertas al mismo tiempo. En realidad, solo un capiLar es perfundido mientras los demás permanecen inactivos ( el $80 \%$ de todos los capilares permanecen cerrados en cualquier momento). Los capilares se abren en forma rotatoria según las necesidades celulares. 
Las células cebadas, secretan Histamina cuando hay anoxia, con lo que se abren los capilares.

Después de una hemorragia o de un trauma, aumentan los niveles de catecolaminas en la sangre produciendo vaso - constricción marcada que puede o no mantener la presión arterial.

Las arteriolas con vasoconstricción dejan pasar menos sangre y entonces se pueden abrix los "Shunts" arteriovenosos, todo lo cual disminuye el flujo a través de la arteriola y el capilar y por consiguiente disminuye la entrega de oxígeno a las células.

Muchos o la mayoría de los capilares pueden permanecer abiertos en un momento dado, en respuesta a una mayor demanda del tiempo de perfusión por las células. Sin embargo, hay menos flujo a través de las arteriolas para suministrar sangre a todos los capilares abiertos y esto produce dos efectos:

a) El espacio vascular es más grnde de lo normal, porque se abren capilares y vénulas. Un volumen sanguíneo normal es entonces insuficiente para llenar todos estos vasos, que no alcanzan a llenar las venas y se produce disminución de la presión venosa central y por consiguiente del retorno venoso.

b) El lento flujo capilar provoca anoxia celular. El metabolismo anaerobio comienza a producir "acido láctico", que no alcanza a ser removido por el flujo lento, lo que produce acidemia lácticavenosa que disminuye el $\mathrm{PH}$. La sangre ácida con flujo lento se hace hipercuagulable.

Se presenta entonces una coagulación intravascular de consumo que bloquea la perfusión capilar produciendo la muerte celular.

Weil Ha producido experimentalmente evidencias de que cuando las endotoxinas entran en el torrente circulatorio, hay obstrucción del flujo venoso hacia el hígado. (43) (44) (5) (7)

Reid Ha obtenido evidencia experimental de que las endotoxinas tienen un efecto vascular periférico. En casos de infección por positivos, el Shock ha sido atribuído a una hipersensibilidad bacteriana, que produce anafilaxis (4)

Evidencias experimentales - Kwoav - Weil Slwch, (5) inyectó conejos con dosis subletales de endotoxinas y comprobó sobrevida de más del $90 \%$ de los animales cuando bloqueaba el plexo celíaco con Xilocaina al 1 - 2\%, vía percutánea.

En los últimos cien años la determinación de la presión sanguínea se ha convertido en una práctica corriente y el descenso de la misma ha llegado a ser signo para diagnóstico y pronóstico del Shock. Datos recientes confirman la opinión de Archibald y M'cleon, quienes en 1917 nos advertían que aunque el descenso de la presión sanguínea es uno de los signos más constantes del Shock, no es un factor esencial y mucho menos su causa. Hemos estado poniendo demasiada atención a la presión sanguínea, tanto que hemos llegado a considerarla inconcientemente como mecanismo causal. Esta tendencia por parte del médico ha conducido en las últimas décadas a usar vasopresores y vasoconstrictores en el tratamiento del Shock.

Estos agentes terapéuticos se han ido desechando al disponerse de nuevas formas de tratamiento derivadas de los nuevos conceptos de los transtornos Fisiológicos del Shock. Hoy se considera que la perfusión tisular y el gasto cardíaco son factores mucho más importantes que la presión sanguínea.

El índice de mortalidad no está en relación con la presión sanguínea, sino con el "grado de acidosis láctica" consecutiva a la Hipoperfusión tisular. La acidemia es proporcional al grado de perfusión tisular.

\section{CAMBIOS FISIOPATOLOGICOS A NIVEL DE ORGANOS}

En el sistema R. Kupffer.

Degeneración de las células de kupffer. Necrosis Focal. Destrucción de macrófagos. Disminución de la resistencia antibacteriana. 


\section{Función Pulmonar}

Cambios de elasticidad. Vasoconstricción. Cambios en $\mathrm{O}_{2}$ y C $\mathrm{C}_{2}$. Acidosis. Hipoxia. Retención de $\mathrm{C}_{2}$. Edema Pulmonar.

\section{En el Tracto Gastrointestinal}

Menos flujo visceral. Necrosis. Hemorragias.

\section{En el Riñón}

Disminuye el flujo plasmático renal. Oligurias y Anurias. Insuficiencia renal aguda. Necrosis tubular renal. Aumento del nitrógeno protéico. Activación del sistema renina-angiotensina.

\section{En el Corazón}

Insuficiencia cardíaca congestiva. Hipoxia Coronaria. Disminuye el gasto cardíaco. Disminuye la presión venosa central. Disminuye la presión arterial. Disminuye el flujo sanguíneo a las extremidades.

\section{En el Hígado}

Menor capacidad para metabolizar el lactato. Menor detoxificación.

\section{En el Pancreas}

Cambios degeneraticos en los accinos. Favorece la autodigestion.

\section{En el Sistema Nervioso Central}

Degeneración Neuronal. Menor actividad en Electro-encefalogramas. Disminuye la tensión Cortical de $\mathrm{O}_{2}$.

\section{En el Metabolismo}

Ph. promedio 7.32

Disminuye el Bicarbonato (13 m. Eq. L). Aumento del lactato promedio (10.2m. Eq. L).

\section{En la Sangre}

Leucopenia. Granulaciones tóxicas. (5) (6)

\section{TRATAMIENTO}

(32) (37) (40) (16)

\section{A. Historia Cinnica completa}

B. Diagnóstico Adecuado

C. Reemplazo adecuado de Líquidos. Un flujo sanguíneo suficiente previene el estado de coagulación intravascular disemi nada.
Hay una mayor demanda de volumen sanguíneo por expansión del lecho vascular por lo cual el volumen normal es inadecuado, debiéndose por tanto, añadir volumen suficiente para llenar los capilares, de manera que haya suficiente volumen venoso de retorno para mantener la presión venosa central.

D. Administración de Antibióticos. Debe iniciarse lo antes posible sin esperar informes de laboratorio. Puede usarse una asociación de Kanamicina o Gentamicina con Cefalotina. Si se comprueba que el germen causal es gram-negativo, el antibiótico de èlección es la Kanamicina, aunque otros consideran que es la Gentamicina; pero debe tenerse en cuenta la función renal. Cuando hay germenes gram-positivos o estos están asociados, se recomienda la Penicilina Cristalina. También pueden usarse 12 gramos por día de Ampicilina intravenosa, o bien Tetraciclinas y Cloranfenicol de acuerdo con los antibiogramas, ya que son drogas bacteriostáticas.

E. Incisión - Drenaje o extirpación del foco infeccioso.

F. Expansores del Volumen Sanguíneo El volumen de la sangre circulante generalmente es inadecuado. Aunque no sea una medida directa del volumen sanguíneo, la Presión venosa Central (P. V. C.), indica la capacidad del corazón para recibir y recircular la sangre; también sirve para determinar el volumen circulatorio óptimo y la velocidad a que debe hacerse la administración de los líquidos. (7) (37)

Si la presión venosa central no es alta, está indicada la administración de líquidos. Aun cuando el volumen sanguíneo no sea bajo, la adıción de líquidos por lo general mejora el rendimiento cardíaco, mientras la Presión Venosa Central no indique una sobrecarga.

La sangre y el plasma son los mejores expansores, pero algunos prefieren las soluciones salinas isotónicas. También se han empleado los dextranos de bajo peso molecular. (Dextran 40 con peso molecular de 40.000) 
G. Corrección de la Acidosis y del desequilibrio de Electrolitos. Debe administrarse Bicarbonato de Sodio o una concentración de alrededor de $100 \mathrm{~m}$. Eq./litro de líquido intravenoso; con dosis inicial$2 \mathrm{~m}$. Eq. $/ \mathrm{kgm}$. de peso.

Deben evitarse soluciones que contengan lactato ya que el hígado no puede motabolizarlo rápidamente para que surta efecto.

H. Heparina. Por la creencia de que la "coagulopatía de consumo" esté presente en el Shock Séptico, se ha usado la Heparina. Sin embargo este tratamiento debe de jarse para los pacientes que tienen una coagulación acelerada y debe practicarse antes de que se produzca una desfibrinación completa, pués de lo contrario se puede incrementar la tendencia hemorrágica (8).

La dosis de Heparina que se recomienda, es de 10.000 unidades que se pueden repetir cada 4 horas si es necesario.

I. Corticorteroides No hay pruebas de que sean beneficiosos en el Shock Séptico y su uso está en controversia; el exacto mecanismo de su acción no está claro. Los que los recomiendan piensan en sus efectos antitóxicos y anti-inflamatorios que restablecen el tono vascular. Disminuyen la resistencia periférica, bloquean los intensos efectos simpático-miméticos de la endotoxina, aumentan la capacidad vascular y el gasto cardíaco. Alivian la relativa insuficiencia Adreno-cortical para el soporte del paciente en Stress y preservan la membrana celular. Se usan intravenosos en grandes dosis, $35-50$ $\mathrm{mg}$ de hidrocortisona por $\mathrm{kg}$ de peso cada 4 horas (33), pero no se recomienda su uso por mas de 48 horas.

J. Vasopresores y Vasodilatadores La vasoconstricción periférica que acompaña al Shock Séptico manifestada por (14) (15) (47) (22) (40)

Piel fría humeda y pálida, cianosis, hipotermia, tiempo de relleno capilar muy prolongado, diferencia de más de $8^{\circ} \mathrm{C}$. entre la temperatura central y la periférica (extremidades), oliguria y anuria, etc., puede causar daños irreversibles en los tejidos insuficientemente perfundidos.

En la actualidad los investigadores clínicos están casi todos de acuerdo en que el tratamiento sensato depende de mejorar la eficiencia del flujo sanguíneo a los órganos vitales y solo en forma secundaria de establecer la presión arterial adecuada.

Estudios recientes confirman que el aumento de la presión arterial no se acompaña necesariamente de incremento del flujo sanguíneo. El término vasopresor es en sí engañoso. Cuando la vasoconstricción es grave y prolongada, algunos clínicos emplean drogas estimulantes adrenérgicas Beta o bloqueadores Alfa.

Este concepto de los Alfa y Beta receptores fué introducido por Ahlquist en 1948 . Es una forma de explicar los hechos y no significa que tales receptores se hayan demostrado anàtómicamente. El autor sugirió que los Alfa-receptores mediaban la respuesta vaso-constrictora en el sitio efector, es decir "son sitios de control a través de los cuales se produce la acción adrenérgica simpática vasoconstrictora”. Los Betareceptores "mediarían la respuesta activa vasodilatadora simpática en los vasos del músculo esquelético". Así se encontraron drogas con efectos betamiméticos y alfamiméticos o combinados.

De estas drogas se puede emplear ya sea un estimulante Beta-adrenérgico, el Isoproterenol o un agente bloqueador adrenérgico Alfa como la Fenoxibenzamina.

Para administrar estos compuestos es necesario corregir la volemia y en lo posible, tener presiones venosas centrales de 12 $16 \mathrm{cms}$. de agua, a causa del mismo efecto vasodilatador de la droga. También deben administrarse expansores de volumen. (Sangre total, Plasma; Soluciones Salinas isotónicas, Dextranos de bajo peso molecular).

Se han usado algunos agentes que reducen el tono vascular por mecanismos no bien conocidos, como la clorpromazina, que tiene también efectos vasodilatadores por acción central, es anti-serotonímico y antihistamínico; se cree que puede ser bloquea- 
dor Alfa-adrenérgico. Esta droga es la que utilizamos en los casos que presentamos en este estudio a dosis de $0.5-1 \mathrm{mg} / \mathrm{kgr}$. de peso en dosis fraccionada por lo menos cada 6 horas. (9) (10)

De acuerdo con el siguiente programa de manejo presentamos un estudio y sus correspondientes resultados en 15 casos, en un período de 3 años.

\section{PLAN DE TRATAMIENTO}

1. Obtención de muestras para cultivos. (Sangre, orina, otros).

2. Disección de la Vena Basílica y paso de cateter R-37 de Baxter hasta los grandes vasos, para tener medida constante de la Presión Venosa Central, lo que es muy importante para la administración de Líquidos.

3. Examenes de Laboratorio:

He mograma

Electrolitos

$\mathrm{Ph}$. Arterial

Examen parcial de Orina

Coagulación y plaquetas

4. Controles continuos:

Presión Venosa Central

Presión Arterial

Pulso

Respiraciones

Temperatura

5. Sonda Vesical para valorar la eliminación urinaria $(0.5 \mathrm{cc}$. por minuto para unos $30 \mathrm{cc}$. por hora).

6. Reemplazo de líquidos

7. Corrección de la acidosis con Bicarbonato de Sódio.

8. Digitalización rápida si es necesaria.

9. Antibióticos (En nuestros casos usamos penicilina cristalina. 24 millones en 24 horas y Clorafenicol 3-6 gm. en 24 horas vía intra-venosa); (por la facilidad de conseguir estas drogas dado su bajo costo).

10. Traqueostomía si es necesario.

11. Oxígeno húmedo permanente (mientras hay vasoconstricción).
12. Heparina (Usamos 6000 unidades cada 6 horas; mientras el paciente está en vasoconstricción y una vez bloqueada la vasoconstricción se suspende.

13. Como-droga de efectos vasodilatadores que ya explicamos, usamos la Clorpro. mazina en dosis de $1 \mathrm{mgr} . / \mathrm{Kg}$, en forma fraccionada según respuesta de la vasoconstricción.

Las causas que produjeron el Shock Bacteriano en los casos analizados fueron:

Aborto Séptico ...... 8 casos

Aminionitis .......4 4 casos

Endometritis ....... 2 casos

Absceso Pélvico - Peritonitis 1 caso

El cultivo en los casos en que se logró aislar gérmenes en la sangre fué positivo para:

Klebsiela-Areobacter en ... 5 casos

E. Coli en ......... 3 casos

Enterococo ......... 1 caso

En el resto de los casos por causas múltiples no fué posible obtener el cultivo.

La edad de las pacientes estuvo comprendido entre los 20 y 41 años, como datos extremos; ya que en este período son más frecuentes las complicaciones del embarazo; (Endometritis, amnionitis, Aborto provocado).

El leucograma en 11 casos estaba dentro de límites normales; en 4 casos había leucocitosis. Esto se puede explicar por lo que ya se ha dicho, de que en la septicemia por gérmenes gram-negativos, el leucograma se puede encontrar dentro de lo normal o con tendencia a la leucopenia.

La presión arterial de las pacientes en el momento de hacer el diagnóstico de Shock Séptico fué:

Presión Arterial de cero (0) ...8 casos Presión Arterial 60/40 m.m. Hg.. . 7 casos

Esto es importante porque muchos autores relacionan los datos de presión arterial con la severidad del proceso; con el tiempo de recuperación y aún con la tasa de mortalidad. En el grupo con Presión Arterial de $0 / 0$ estaban las pacientes que llegaron al Hospital en Shock severo. 
La presión arterial una vez recuperada la paciente fluctuó entre $90 / 60 \mathrm{~m} . \mathrm{m}$. Hg. y 120/80 m.m. Hg.

El tiempo de latencia de la respuesta al tratamiento, es decir el tiempo que transcurrió hasta la recuperación de la paciente del Shock Séptico una vez iniciado el programa de manejo ya explicado, se repartió así:

De 1-6 horas .......... 3 casos

De $6-12$ horas ........... 2 casos

De $12-18$ horas .......... 3 casos

De $18-24$ horas.......... 5 casos

De mas de 24 horas.. . . . . . 2 casos

Es de anotar que la mayoría de las pacientes ingresaron al Hospital ya con el Shock establecido muchas horas antes de la consulta.

El período de observación hospitalaria después de la recuperación de la paciente fué de 2 a 10 días.

Como complicaciones ocurridas durante la observación del grupo de casos analizados podemos mencionar:

1. Flebitis en Miembros inferiores y superiores: En dos (2) casos (Cateteres? ).

2. Moniliasis Gastrointestinal: En un (1) caso.

Es de anotar que ninguna paciente tuvo como secuela la insuficiencia renal aguda que era bastante común antes de iniciar este plan de tratamiento. Esto se puede explicar por las mismas bases del manejo tendientes a bloquear la vasoconstricción.

Otro hecho importante de anotar es que varias de las pacientes del grupo que se presenta, antes de este plan terapéutico habían recibido altas dosis de drogas del tipo Aramine, Levofed y Presorol sin obtener resultados satisfactorios; y se incluyeron en el estudio, por considerar un fracaso la terapia convasopresores.

Más del 50\% de los casos han tenido controles posteriores en la Consulta Externa del Hospital Universitario, por períodos de 6 meses a 2 años, con magnífica evolución y sin secuelas aparentes.

Del grupo estudiado solo tuvimos una (1) muerte; en una paciente que habíamos lo- grado recuperar del Shock, ya que al momento de la muerte tenía presiones arteriales de $120 / 80 \mathrm{~m} . \mathrm{m}$. Hg., sin signos de vasoconstricción. Creemos que la causa de la muerte fué la de múltiples embolias sépticas a órganos vitales. (Tuvo una muerte súbita), cuando la creíamos muy recuperada, al 60. día de tratamiento. No fué posi ble la autopsia por oposición de la familia.

\section{RESUMEN Y CONCLUSIONES}

1. Se hacen una serie de consideraciones históricas, etiológicas y fisiopatológicas del "Shock Séptico, especialmente por gérmenes gram-negativos.

2. Se analiza un plan de tratamiento basado en el manejo adecuado de líquidos y electrolitos, antibióticos, Oxígeno húmedo, digitalización, monitorias constantes de la presión arterial, pulso, presión venosa central, respiraciones, temperatura y eliminación urinaria.

3. Se analiza el concepto de los receptores Alfa y Beta y las drogas que los activan o inhiben bloqueando su acción, o estimulandola.

4. Se presenta el análisis, estudio y tratamiento de 15 casos según plan de tratamiento establecido previamente.

5. Se presenta un porcentaje significativo de casos de Shock Séptico como complicación del Aborto Séptico. (más del 50\%).

6. Se anota el hallazgo persistente y. en buena proporción de gérmenes gramnegativos como la klebsiela - Areobacter y E. Coli.

7. Los datos de presión arterial en el momento del diagnóstico; más del 50\% con cero (0) de Presión Arterial, corresponden a las pacientes que al ingresar al Hospital estaban en Shock severo.

8. El tiempo de recuperación de las pacientes una vez iniciado el plan terapéutico fue en más del $66 \%$ de los casos de 12 a 24 horas. 
9. Las complicaciones y secuelas fueron mínimas: flebitis superficial en dos (2) casos, moniliasis gastrointestinal en un (1) caso.

10. Sugerimos el uso de drogas con efectos vasodilatadores que bloquean la vasoconstricción, evitan do el daño celular y la muerte, en los casos de Shock Séptico con "vaso constricción severa".

11. Por analisis de las Historias del Hospital Universitario del Valle, en un período de 10 años encontramos mortalidad por Shock Séptico de más del $76 \%$ de los casos, (Con tratamiento a base de vasopresores).

En el grupo que presentamos a consideración tenemos una mortalidad del $7 \%$, lo cual habla por sí mismo de las bondades del tratamiento establecido en estos casos.

12. Recomendamos de acuerdo con muchos autores, el plan terapéutico expuesto en este trabajo cuando se ha establecido claramente el diagnóstico del Shock Séptico con evidencia clínica de "vasoconstricción".

\section{SUMMARY AND CONCLUSIONS}

1. Several historical, ethiological and physio-pathological considerations are issued, mainly on "Septic Shock"

2. A treatment Plan is analyzed, based on the adequate handling of liquids and electrolites, antibiotics, wet exygen, digitalization, continuous monitoring of blood pressure, central venous pressure, respiratory frequency, temperature and urine elimination.

3. The concept of the Alfa and Beta receptors is analyzed, together with the drugs which activate or inhibit their action by blocking or estimulating it.
4. The analytical study and treatment of 15 cases according to a pre-established treatment plan, is shown.

5. A significant percentage of Septic Shock cases as a complication of Septic Abortion is shown, (more than $50 \%$ ).

6. The persistent discovery of GramNegative germs such as klebsiella Aerobacter and E. Colli etc., in large amounts is observed.

7. Data on blood pressure at the time when diagnosis was made, more than $50 \%$ with 0 , corresponding to patients in Septic Shock at the time of arrival at the Hospital.

8. Recovery time of patients once the therapeutic treatment started: from 12 to 24 hours in more than $66 \%$ of the cases.

9. Complications and sequelas were minimal: - slight flebitis in two cases, gastrointestinal moniliasis in one case.

10. We suggest the use of drugs with a vasodilatory effect to block vasoconstriction thus avoiding cellular damage and death in case of Septic Shock with severe vasoconstricción.

11. Analyzing clinical histories at the "Hospital Universitario del Valle", in a ten year period we found $76 \%$ mortality in Septic Shock cases (treated basically with vasopressors). Within the group we are showing we have $70 \%$ mortality, a self-explanatory figure of the benefitial effects of the treatment followed for those cases.

12. In agreement with several authors, we recommend the therapeutic treatment explained in this report when a Septic Shock diagnosis has been clearly made with clinical evidence of vasoconstricción conditions. 


\section{REFERENCIAS}

1. A. Bane. Surg. Gynec. Obstet. 127:4. Octubre 1968.

2. Adams R. H.; and Pritchard, J. A. "Bacterial Shock in Obstetrics and Gynecology" Obst. and Gynecology 16: $387 ; 1960$.

3. Adcock, L.L., and Hakanson E.Y. "Vascular Collapse in Septic Abortion".

4. Advances in Internal Medicin. 17:363 1971.

5. Ann. Surg. 165.: 4; $504-518-536$ Abril 1967.

6. Anuario de actualización en Medicina IMSS: Fasc. A. Cardio-vascular: 1969.

7. Berk, J.M. Et Al the Treatment of endotoxin Shock by Beta Adrenergic Blockade. Ann of Surg. 169: 74, 1969.

8. Berck, J. L. Et Al. The role of Beta Adrenergic Blockade in the treatment of Séptic Shock. Surg. Ginec. and Obst. 130: 1025. 1970.

9. Carta Médica 11: 33. Abril 19-1969.

10. Carta Médica 11:23 Nov. 14 de 1969 (Corresponde a ed. 283 de the medical Letter).

11. Cavanagh. D. Am. J. Obstet. - Gynec. 96: 7.913.

12. Clemetson, C.A., Moshfeghi, M.M. Strange effect of Dextrose 5\% en in Water. J. Med. 280:332. 1969. New Eng.

13. Collins. V. J. et al. The use of Chol promazine in Shock Surg Clin. N. Amer. 44: 173 - 183 - 1964.

14. Collins, E.J.: Newer attitudes in the management of Hemorrhagic Shock. The use of Clorpromazine as adjunnet. Surg. Clin. N. Americ. 44: 173 1964.
15. Denis CA Vanag. H.M.D.; Allan G. W.M. Levd. Septic. Shock in Obstetrics and Gynecology American Journal. Obst. And Gynec. 96, Number 7, December 1966.

16. Douglas, G. Am. J. Obstet, Gynec. 96: 5 Nov. 1966.

17. Fisiopatología y tratamiento del Scho ck. Jama 199: 11; 115 Marzo 1967.

18. Freeg; Gor Duer; Geitmann. American Journal Obst. Gynec. Julio 1960: 104.

19. Freel, et al Gorduer, Geihman. Amecan Journal. Obst. Gynec. Julio 1969. 104

20. Fromm. S. et. al Phenoxy - benza mine in Human Shock. Surg. Gynec. and Obst. 129: 4 Oct. 1969.

21. Ginsburg. J. Physiology and Management of severe Shock Obst. Gynec. Survey . 21: 730, 1966.

22. Gram Negative Bacteremia Shock Jama 207,: 2.333, January 131969.

23. Han Ming Kwaan and Harry Weil. Differences in the Mechanism of Shock caused by Bacterial Infections. Sugery Gynecology and Obstetric. 128: 1 January 1969.

24. Hardaway. Jamma 99: 11 marzo 13 1967.

25. Hemodynamic Studies and Results of Therapy in 50 patients with Bacteremic Shock. The American Journal of Medicin 54:421. April 1973.

26. Hemorragic and Endotoxic Shock $\lambda$. Pathophysiologic approach. To Diagnosis and Management. American Journal of Obstetrics and Gynecology. 110: 8; 1041. August 13. 1971.

27. J. Robins. Obstet. Gynec. 28: 1130 Julio 1966.

28. Jacob Fine. Jama 188: 5 Mayo 4 1964: 427.

29. Jama 199: 11. 115 Marzo 1967.

30. Kwoav - Wcil: Slwxh. Surg. Gynec Obst. Enero 1969. 128. 
31. Lillehei, R.C. and Gynecology 29, Nomberg, June 1967: 806.

32. Leon L. Adock, Erik y Hakanson. Vascular collapse complicating septic abortion. American Journal of Obst. and Gynec 79: 516. 1960

33. M.H. Weil et. al. Jama. 192: 668, 1965.

34. Martinez, J. T., Fernandez, V. Leon, $\mathrm{H}$. Clinical evaluation of new therapeutic concepts in septic Shock. Obst and Gynec 79: 516. 1960.

33. M.H. Weil et. al. Jama. 192: 668, 1965.

34. Martinez, J. T., Fernande z, V. Leon, H. Clinical evaluation of new therapeutic concepts in septic Shock. Obst and Gynec. 27: 296. 1966.

35. Mc' Arthur, J. W. New Englad. J. Med. 261: 461, 1969.

36. Murphy Gerald P. et. al Benson Donald W. Schrimer, Horst. K. A. Renal response to Clorpromazine in hemorragic., Hipotensión, Hemodynamic, and metabolic chances and adrenolytic efectin dogs. Annals surgery 164: 867. 1966.

37. Obstetrics and Gynecology 29: $6 \mathrm{Ju}-$ ne 1967: 806 .

38. R.W. Anderson P.M. James et. al. Bredemberg and M. Hardaway. Division of Sugery Walter Reed. Army Medical Center Washinton distrit of
Co. - Phenoxybenzamine in septic Shock Ann. Surg. 165: $341-350$ March. 1967.

39. R. W. Evans et al Am. J. Med., 46: 910, Junio 1969.

40. Reilly D. J. Klimpel; D. Cohon, M. S. Heller, W. M.: Kossler, A. M. Kautz, H. D.: PH. intravenous solutions. New Eng. J. Med. 280: 900902 - 1969.

41. Shock: Anuario actualización Médica. Fasc. (9), cardiovascular IMSS 1969 México.

42. Shock Endotoxico Bacteriano en Pediatría. La prensa Médica Argentina 58: 25. Pag. 1269, Agosto 20-1971.

43. Sindrome de Shock. Acta Médica Venezolana 16: 9-10: Sept. Oct. 1969.

44. Survey of anesthesiology. December 1967. 11: 6 .

45. The treatment of endotoxin by Beta Adrenergic blokade. Annals of surgery. January 1969. 169: 1.

46. The reversibility of irreversible Shock Lancet. 2: 1365, 1963.

47. The use of P.V.C. on accurate for body Fluid replacement Surg. Gynec. Obst. 1965, 120: 545.

48. Weil, M.H. Endotoxin Shock. Clinic. Obst, Gynec. 4: 971 - 1963.

49. Weil, Shubin. Ann Int. Med 60: 3; 384, Marzo 1964. 